\title{
Über das spezifische Drehungsvermögen einiger aus Pflanzen dargestellten Tyrosinpräparate.
}

\author{
Von \\ E. Schulze und E. Winterstein.
}

(Aus dem agrikultur-chemischen Laboratorium des Polytechnikums in Zürich.)

(Der Redaktion zugegangen am 28. April 1905.)

In einer vor drei Jahren publizierten Abhandlung ${ }^{1}$ ) teilten wir Resultate mit, die bei der Untersuchung einiger aus den Keimpflanzen von Cucurbita Pepo und aus Kartoffelknollen dargestellten Tyrosinpräparate erhalten worden waren. Diese Präparate zeigten ein relativ hohes spezifisches Drehungsvermögen; als wir ihre Lösung in $4 \%$ iger Salzsäure im Soleil-V entzk eschen Polarisationsapparat untersuchten, wurde $[\alpha]^{{ }^{16}}$ gleich $-14,6$ bis - 16,10 gefunden, während Emil Fischer ${ }^{2}$ ) bei Anwendung des gleichen Lösungsmittels für ein durch Spaltung von Casein durch Salzsäure dargestelltes Tyrosinpräparat $[\alpha]^{D^{20}}=-12,56^{\circ}$, für ein zweites Präparat $[\alpha]^{D^{20}}=-13,2^{0}$ fand (ein noch niedrigeres Resultat, nämlich $[\alpha]^{\mathrm{D}^{16}}=-11,6^{0}$ erhielten wir bei Untersuchung von Tyrosin, welches ebenfalls durch Spaltung einer Eiweißsubstanz mittels Salzsäure gewonnen worden war). Bei Mitteilung jener Resultate wiesen wir aber darauf hin, daß Emil Fischer (loc. cit.) bei der Spaltung von racemischem Tyrosin in die optisch aktiven Komponenten ein rechtsdrehendes Präparat erhalten hat, für welches bei Anwendung des gleichen Lösungsmittels $[\alpha]^{\mathrm{D}^{20}}=+16,4^{0}$ gefunden wurde; dieses Präparat drehte also noch etwas stärker nach rechts, als das von uns aus Kartoffelknollen dargestellte Tyrosin nach links. Für diese Differenzen würde man, wie an jener Stelle von uns aus-

1) Diese Zeitschrift, Bd. XXXV, S. 299.

2) Ber. d. Deutsch. chem. Ges., Bd. XXXII, S. 3646. 
gesprochen wurde, eine Erklärung haben, wenn man annehmen könnte, daß den bei der Spaltung von Eiweißstoffen durch Säuren erhaltenen Tyrosinpräparaten racemisches Tyrosin in wechselnder Quantität beigemengt ist.

In unserer Abhandlung teilten wir mit, daß es in unserer Absicht liege, aus den Knollen von Dahlia variabilis, einem an Tyrosin relativ reichen pflanzlichen Objekt, noch ein Präparat dieser Aminosäure darzustellen und auf sein spezifisches Drehungsvermögen zu untersuchen. Dahliaknollen, die wir im Frühjahr 1903 verarbeiteten, gaben eine sehr geringe Ausbeute an Tyrosin; besser war die Ausbeute aus Knollen, die wir im folgenden Jahre untersuchten. Die Darstellung des Tyrosins aus letzteren geschah in folgender Weise: Die zerriebenen Knollen wurden mit kochendem 80\% igen Alkohol behandelt. Das durch Filtration vom Ungelösten getrennte Extrakt unterwarfen wir der Destillation; der dabei erhaltene Rückstand wurde in Wasser aufgenommen, die Lösung zur Reinigung zuerst mit Tannin, später noch mit Bleiessig versetzt. Dem Filtrat vom Bleiniederschlag wurde Mercurinitrat in schwachem Überschuß, sodann zur Abstumpfung der Säure noch etwas Natriumkarbonat zugefügt. Die dadurch erzeugte Fällung wurde nach Verlauf einiger Tage abfiltriert, ausgewaschen, dann in Wasser verteilt und durch Schwefelwasserstoff zersetzt. Das Filtrat vom Schwefelquecksilber lieferte, nachdem es mit Ammoniak neutralisiert und sodann im Wasserbade stark eingeengt worden war, eine aus Tyrosin bestehende Ausscheidung. Da dieses Produkt noch ziemlich stark gefärbt war, so lösten wir es in verdünnter Ammoniakflüssigkeit und fügten der Lösung unter Umschütteln etwas Bleiessig zu. In den dadurch hervorgebrachten Niederschlag ging neben einem kleinen Teile des Tyrosins die färbende Substanz ein. Die von diesem Niederschlag abfiltrierte Flüssigkeit wurde durch Zusatz von etwas Schwefelammonium vom Blei befreit, nach der Filtration im Wasserbade eingeengt und sodann unter eine Glasglocke über konzentrierte Schwefelsäure gestellt. Das Tyrosin schied sich nach und nach aus der Flüssigkeit aus. Durch Umkristallisieren aus Wasser unter Zusatz von etwas Ammoniakflüssigkeit noch weiter gereinigt, bildete es eine 
aus glänzenden nadelförmigen Kristallen bestehende Masse. Im Kapillarröhrchen schmolz es gleichzeitig mit einem Tyrosinpräparat anderer Herkunft. Die Stickstoffbestimmung lieferte folgende Resultate:

1. $0,1858 \mathrm{~g}$ Substanz (bei $100^{\circ}$ getrocknet) gaben $13,4 \mathrm{ccm}$ Gas bei $713 \mathrm{~mm}$ Druck und $16^{\circ} \mathrm{C} .=0,01464 \mathrm{~g}$ oder $7,88 \% \mathrm{~N}$.

2. $0,2402 \mathrm{~g} \mathrm{Substanz}$ (bei $100^{\circ}$ getrocknet) gaben $17,0 \mathrm{ccm}$ Gas bei $710 \mathrm{~mm}$ Druck und $16^{\circ} \mathrm{C} .=0,01857 \mathrm{~g}$ oder $7,71 \% \mathrm{~N}$.

Im Mittel wurden also $7,80 \% \mathrm{~N}$ gefunden, während die Theorie $7,73^{\circ} / 0$ verlangt.

Die Untersuchung des aus den Dahliaknollen dargestellten Tyrosins im Soleil-Ventzkeschen Polarisationsapparat gab folgendes Resultat:

Eine Lösung in $4 \%$ iger Salzsäure, welche in $25 \mathrm{ccm}$ 2,2676 g wasserfreie Substanz enthielt, drehte im $200 \mathrm{~mm}$-Rohr bei $20^{\circ}$ C. $6,8^{\circ}$ nach links; demnach ist $[\alpha] \mathrm{D}^{20}=-12,9^{\circ}$.

Die salzsaure Lösung des für diese Bestimmung verwendeten Tyrosinpräparates wurde nun mit Ammoniak genau neutralisiert, das dabei sich ausscheidende Tyrosin später abfiltriert, mit kaltem Wasser gut ausgewaschen, dann getrocknet und wieder im Polarisationsapparat untersucht. Wir erhielten nun folgendes Resultat:

Eine Lösung in 4\% iger Salzsäure, welche in $25 \mathrm{ccm}$ $2,0910 \mathrm{~g}$ wasserfreie Substanz enthielt, drehte im $200 \mathrm{~mm}$-Rohr bei $20^{\circ}$ C. $6,1^{\circ}$ nach links; demnach ist $[\alpha]^{20}=-12,5^{\circ}$.

Die Differenz zwischen dieser und der im ersten Versuch gefundenen Zahl liegt innerhalb der Fehlergrenze unserer Bestimmungen. Wie man sieht, weichen diese Zahlen nur wenig von denjenigen $a b$, welche E. Fischer bei Bestimmung des spezifischen Drehungsvermögens von zwei durch Spaltung einer Eiweißsubstanz mit Salzsäure erhaltenen Tyrosinpräparaten erhielt. (Diese Zahlen sind oben schon angegeben worden.)

Wir bestimmten ferner noch das spezifische Drehungsvermögen eines Tyrosinpräparates, welches bei der Autolyse junger Keimpflanzen von Lupinus albus erhalten worden war. Dieses Präparat war durch Auflösen in verdünnter Salpetersäure und Wiederausfällen mit Ammoniak, sowie durch Umkristallisieren 
aus Wasser unter Zusatz von etwas Ammoniakflüssigkeit gereinigt; es bestand aus weißen glänzenden Kristallnadeln. Die Untersuchung der salzsauren Lösung im Soleil-Ventzkeschen Polarisationsapparat lieferte folgendes Resultat:

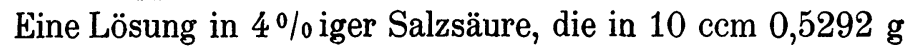
wasserfreie Substanz enthielt, drehte bei $16^{\circ} \mathrm{C}$. im $200 \mathrm{~mm}$-Rohr $5,0^{\circ}$ nach links; demnach ist $[\alpha] \mathrm{D}^{16}=-16,2^{\circ}$.

Von den aus Pflanzen gewonnenen Tyrosinpräparaten, die von uns untersucht worden sind, besaß also nur das aus Dahliaknollen dargestellte ein spezifisches Drehungsvermögen, ${ }^{1}$ ) wie es auch für das durch Spaltung von Eiweißstoffen mit Säuren erhaltene Tyrosin gefunden wurde; die drei anderen Präparate zeigten ein höheres Drehungsvermögen. Es ist nun die Frage zu stellen, ob diese drei Präparate noch Beimengungen enthielten und ob durch letztere das Drehungsvermögen erhöht wurde. Bestimmte Anhaltspunkte für eine solche Annahme liegen nicht vor. Jene drei Präparate glichen im Aussehen vollkommen dem gewöhnlichen Tyrosin; für zwei Präparate wurde ferner konstatiert, daß ihr Schmelzpunkt mit demjenigen des Tyrosins übereinstimmte. Auch wurde durch die Analyse festgestellt, daß das aus Kartoffelknollen dargestellte Präparat den Stickstoffgehalt des Tyrosins besaß (die beiden anderen Präparate sind nicht analysiert worden).

Falls man nun nicht anzunehmen hat, daß jene drei Präparate noch Beimengungen einschlossen, so muß man wohl zur Erklärung ihres stärkeren Drehungsvermögens auf die oben schon ausgesprochene Annahme zurückkommen, daß den bei der Spaltung von Eiweißstoffen durch Säuren erhaltenen Präparaten racemisches Tyrosin beigemengt ist, und daß dadurch ihr Drehungsvermögen herabgedrückt wird. Auch zur Erklärung des relativ niedrigen Drehungsvermögens des aus Dahliaknollen erhaltenen Tyrosins würde man anzunehmen haben, daß dem-

1) Allerdings hatten wir auch ein stärker drehendes Tyrosinpräparat aus Dahliaknollen unter Händen (Diese Zeitschrift, Bd. XXXV, S. 311); da dasselbe jedoch noch gefärbt war und da infolge davon der genauen Feststellung seines Drehungsvermögens Schwierigkeiten entgegenstanden, so konnten wir auf diese Beobachtung keinen Wert legen. 
selben entweder racemisches oder rechtsdrehendes Tyrosin in geringer Menge beigemischt ist. Eine solche Annahme kann nicht als eine unzulässige bezeichnet werden; denn es sind von früher her schon Fälle bekannt, in denen einer optisch aktiven Stickstoffverbindung, die aus pflanzlichem oder tierischem Material abgeschieden wurde, eine kleine Quantität von racemischer oder von entgegengesetzt drehender Substanz gleicher Art beigemengt war. ${ }^{1}$ )

1) Bezügliche Angaben haben wir in dieser Zeitschrift, Bd. XXXV, S. 300, gemacht. $\mathrm{Zu}$ erwähnen ist hier auch, daß nach F. Ehrlich (Ber. d. Deutsch. chem. Ges., Bd. XXXVII, S. 1820) ein aus Melasseschlempe dargestelltes Leucinpräparat neben Isoleucin r-Leucin und I-Leucin einschloß und daß 0. E. v. Lippmann aus Rübensaft ein in salzsaurer Lösung rechtsdrehendes Tyrosin gewann. 